\title{
Adaptive Synchronization of Complex Networks with Mixed Probabilistic Coupling Delays via Pinning Control
}

\author{
Jian-An Wang \\ School of Electronics Information Engineering, Taiyuan University of Science and Technology, Shanxi 030024, China \\ Correspondence should be addressed to Jian-An Wang; wangjianan588@163.com
}

Received 26 February 2014; Accepted 22 June 2014; Published 15 July 2014

Academic Editor: Qing-Wen Wang

Copyright (C) 2014 Jian-An Wang. This is an open access article distributed under the Creative Commons Attribution License, which permits unrestricted use, distribution, and reproduction in any medium, provided the original work is properly cited.

\begin{abstract}
The problem of synchronization for a class of complex networks with probabilistic time-varying coupling delay and distributed time-varying coupling delay (mixed probabilistic time-varying coupling delays) using pinning control is investigated in this paper. The coupling configuration matrices are not assumed to be symmetric or irreducible. By adding adaptive feedback controllers to a small fraction of network nodes, a low-dimensional pinning sufficient condition is obtained, which can guarantee that the network asymptotically synchronizes to a homogenous trajectory in mean square sense. Simultaneously, two simple pinning synchronization criteria are derived from the proposed condition. Numerical simulation is provided to verify the effectiveness of the theoretical results.
\end{abstract}

\section{Introduction}

During the past few decades, synchronization in complex networks has gained increasing research attention [1-7]. There are many different kinds of methods in the study of network synchronization behavior such as adaptive feedback control [8-10], impulsive control [11, 12], passive method [13, $14]$, intermittent control $[15,16]$, and sampled-data control [17-19].

As we know, since the real-world complex networks usually have a large number of nodes, it is impossible to realize network synchronization by adding controllers to all nodes. To reduce the number of controlled nodes, pinning control, in which some local feedback controllers are only applied to a fraction of network nodes, has been introduced in many works [20-29]. Pinning control is an effective synchronization strategy because it is easily realized in practice. In [20], the authors found that one can pin the linearly coupled networks by introducing fewer locally negative feedback controllers. They also found out that the pinning strategy based on highest connection degree has better performance than totally randomly pinning. Chen et al. in [21] pinned a complex network to a homogenous solution by a single controller under a large enough coupling strength. By using adaptive pinning control method, the authors in
[22] investigated local and global pinning synchronization of complex networks and presented some low-dimensional pinning synchronization criteria. In [23], Yu et al. showed that the nodes with low degrees should be pinned first when the coupling strength is small, which is different from the traditional results. The authors in [24] considered the pinning synchronization of a complex network with nonderivative and derivative coupling. Song and Cao in [25] presented some low-dimensional pinning schemes for global synchronization of both directed and undirected complex networks and proposed specifically pinning schemes to select pinned nodes by investigating the relationship among pinning synchronization, network topology, and the coupling strength. Furthermore, Song et al. in [26] investigated the pinning controlled synchronization of a general complex dynamical network with discrete-delay coupling and distributed-delay coupling. Some sufficient conditions for the synchronization to require the minimum number of pinning nodes were derived in [27], and the method for calculating the number of pinning nodes was given by using the decreasing law of maximum eigenvalues of the principal submatrixes. Recently, the pinning sampled-data synchronization problem was addressed in [28].

Time delay is ubiquitous in many physical systems due to the finite switching speed of amplifiers, finite signal 
propagation time in biological networks, memory effects, and so on. In order to give a more precise description of dynamical network, time delay should be considered inevitably. Therefore, much effort has been devoted to the study of the synchronization of complex networks with coupling delays. It is worth pointing out that, among most existing results, the network synchronization problem has been predominantly studied for complex networks with deterministic delays. However, as reported in [30], the probability distribution of time delay in an interval is an important characteristic in networked control systems [30]. The probability of the delay appearing in lower interval is large and long delay happens with a low probability, which will lead to some conservatism if only the information of variation range of time delay is considered. Thus, coupling delay in complex networks may exist in a random form and take values according to probability in different interval ranges [31]. In addition, it is noted that time delays can be generally categorized as discrete ones and distributed ones. Moreover, it has been observed that they usually have a spatial nature due to the presence of a number of parallel pathways of a variety of axon sizes and lengths in a network. To the best of the authors' knowledge, up to now, little attention has been paid to the study of pinning synchronization problem for complex networks with probabilistic time-varying coupling delay and distributed time-varying coupling delay, which motivates our investigation.

In this paper, we are concerned with the synchronization problem in an array of hybrid-coupled complex networks with mixed probabilistic time-varying coupling delays by pinning control scheme. The coupling configuration matrices are not assumed to be symmetric or irreducible. Under a low-dimensional condition, the network can be asymptotically pinned to a homogenous state in mean square sense by applying adaptive feedback control actions to a small fraction of nodes. Also, two pinning synchronization criteria are obtained for simple cases. A numerical example is given to demonstrate the effectiveness of the proposed results.

The rest of this paper is organized as follows. In Section 2, the model of complex dynamical network with mixed probabilistic time-varying coupling delays is presented and some preliminaries are also provided. Pinning adaptive synchronization criterion is discussed in Section 3. Numerical simulations are given in Section 4. Finally, a conclusion is presented in Section 5.

Notations. $R^{n}$ and $R^{m \times n}$ denote the $n$-dimensional Euclidean space and the set of all $m \times n$ real matrices, respectively. The superscript " $T$ " represents the transpose, and " $I$ " denotes the identity matrix with appropriate dimensions. $\operatorname{diag}\left\{l_{1}, l_{2}, \ldots, l_{n}\right\}$ stands for a block diagonal matrix. The notation $A \otimes B$ represents the Kronecker product of matrices $A$ and $B . \lambda_{\text {min }}(A)$ and $\lambda_{\text {max }}(A)$ are the minimum and the maximal eigenvalue of symmetric matrix $A$, respectively. $G_{l}$ denotes the minor matrix of $G$ by removing its first $l$ rowcolumn pairs. $E\{\cdot\}$ is the mathematical expectation.

\section{Preliminaries and Model Description}

Consider a complex dynamical network consisting of $N$ identical nodes, which is characterized by

$$
\begin{aligned}
\dot{x}_{i}(t)= & f\left(x_{i}(t)\right)+c_{1} \sum_{j=1}^{N} g_{i j} \Gamma x_{j}(t) \\
& +c_{2} \sum_{j=1}^{N} a_{i j} \Gamma x_{j}(t-\tau(t)) \\
& +c_{3} \sum_{j=1}^{N} b_{i j} \Gamma \int_{t-r(t)}^{t} x_{j}(\xi) d \xi+u_{i}, \quad i=1,2, \ldots, N,
\end{aligned}
$$

where $x_{i}=\left(x_{i 1}, x_{i 2}, \ldots, x_{i n}\right) \in R^{n}$ and $u_{i}(t) \in R^{n}$ are, respectively, the state variable and the control input of the $i$ th node. $f: R^{n} \rightarrow R^{n}$ is a continuous vector-valued function. The positive constants $c_{i}(i=1,2,3)$ are the strengths for the constant and delayed coupling, respectively. $\tau(t) \in\left[0, \tau_{2}\right]$ and $r(t) \in[0, r]$ are the discrete delay and distributed delay, respectively. $\Gamma>0$ is the inner coupling matrix between nodes. $G=\left(g_{i j}\right) \in R^{N \times N}, A=\left(a_{i j}\right) \in R^{N \times N}$, and $B=\left(b_{i j}\right) \in$ $R^{N \times N}$ are the coupling configuration matrices. If there is a connection between node $i$ and node $j(i \neq j)$, then $g_{i j}>0$, $a_{i j}>0$, and $b_{i j}>0$; otherwise, $g_{i j}=0, a_{i j}=0$, and $b_{i j}=0$. The diagonal elements of matrices $G, A$, and $B$ are defined as $g_{i i}=-\sum_{j=1, j \neq i}^{N} g_{i j}, a_{i i}=-\sum_{j=1, j \neq i}^{N} a_{i j}$, and $b_{i i}=$ $-\sum_{j=1, j \neq i}^{N} b_{i j}$, respectively. Clearly, in this paper, the coupling configuration matrices $G, A$, and $B$ may be different from each other. Furthermore, $G, A$, and $B$ are not assumed to be symmetric or irreducible.

To describe the complex network model more precisely, the probability distribution of the coupling delay should be employed. Consider the information of probability distribution of the coupling time delay $\tau(t)$; two sets and functions are defined:

$$
\begin{gathered}
\Omega_{1}=\left\{t: \tau(t) \in\left[0, \tau_{1}\right)\right\}, \\
\Omega_{2}=\left\{t: \tau(t) \in\left[\tau_{1}, \tau_{2}\right]\right\}, \\
\tau_{1}(t)= \begin{cases}\tau(t), & \text { for } t \in \Omega_{1}, \\
\bar{\tau}_{1}, & \text { for } t \in \Omega_{2},\end{cases} \\
\tau_{2}(t)= \begin{cases}\tau(t), & \text { for } t \in \Omega_{2}, \\
\bar{\tau}_{2}, & \text { for } t \in \Omega_{1},\end{cases}
\end{gathered}
$$

where $\tau_{1} \in\left[0, \tau_{2}\right], \bar{\tau}_{1} \in\left[0, \tau_{1}\right)$, and $\bar{\tau}_{2} \in\left[\tau_{1}, \tau_{2}\right]$. It is obvious that $\Theta_{1} \cup \Theta_{2}=R^{+}$and $\Theta_{1} \cap \Theta_{2}=\emptyset$. Furthermore, from the definitions of $\Omega_{1}$ and $\Omega_{2}$, it can be seen that $t \in \Omega_{1}$ means that the event $\tau(t) \in\left[0, \tau_{1}\right)$ happens, and $t \in \Omega_{2}$ means that the event $\tau(t) \in\left[\tau_{1}, \tau_{2}\right]$ happens. Then, a stochastic random variable $\beta(t)$ can be defined as

$$
\beta(t)= \begin{cases}1, & t \in \Omega_{1} \\ 0, & t \in \Omega_{2} .\end{cases}
$$


Assumption 1. $\beta(t)$ is a Bernoulli distributed sequence with

$$
\begin{aligned}
& \operatorname{Prob}\{\beta(t)=1\}=E\{\beta(t)\}=\beta_{0} \\
& \operatorname{Prob}\{\beta(t)=0\}=1-E\{\beta(t)\}=1-\beta_{0},
\end{aligned}
$$

where $0 \leq \beta_{0} \leq 1$ is a constant and $E\{\beta(t)\}$ is the expectation of $\beta(t)$.

Remark 2. The Bernoulli distributed sequence $\beta(t)$ is used to describe the randomly varying delay. From Assumption 1, it can be shown that $E\left\{\beta^{2}(t)\right\}=\beta_{0}, E\left\{(1-\beta(t))^{2}\right\}=1-\beta_{0}$, and $E\{\beta(t)(1-\beta(t))\}=0$.

By using the new functions $\tau_{1}(t), \tau_{2}(t)$, and $\beta(t)$, the system (1) can be written as

$$
\begin{aligned}
\dot{x}_{i}= & f\left(x_{i}(t)\right)+c_{1} \sum_{j=1}^{N} g_{i j} \Gamma x_{j}(t) \\
& +\beta(t) c_{2} \sum_{j=1}^{N} a_{i j} \Gamma x_{j}\left(t-\tau_{1}(t)\right) \\
& +(1-\beta(t)) c_{2} \sum_{j=1}^{N} a_{i j} \Gamma x_{j}\left(t-\tau_{2}(t)\right) \\
& +c_{3} \sum_{j=1}^{N} b_{i j} \Gamma \int_{t-r(t)}^{t} x_{j}(\xi) d \xi+u_{i}, \quad i=1,2, \ldots, N .
\end{aligned}
$$

The isolated node of network (1) is given by the following node dynamics:

$$
\dot{s}(t)=f(s(t)) .
$$

Here, $s(t)$ may be an equilibrium point, a periodic orbit, or even a chaotic orbit.

To reduce the number of controllers, we adopt the pinning control approach to synchronize network (5), which means that the control actions are only added to a small fraction $\delta(0<\delta \ll 1)$ of the total network nodes and most of network nodes are not directly controlled. Suppose that the nodes $i_{1}, i_{2}, \ldots, i_{l}$ are selected to be pinned, where $l=\lfloor\delta N\rfloor$ represents the integer part of the real number $\delta N$. Without loss of generality, rearrange the order of nodes and let the first $l$ nodes be controlled. Then we have the following pinning controlled network:

$$
\begin{aligned}
\dot{x}_{i}= & f\left(x_{i}(t)\right)+c_{1} \sum_{j=1}^{N} g_{i j} \Gamma x_{j}(t) \\
& +\beta(t) c_{2} \sum_{j=1}^{N} a_{i j} \Gamma x_{j}\left(t-\tau_{1}(t)\right) \\
& +(1-\beta(t)) c_{2} \sum_{j=1}^{N} a_{i j} \Gamma x_{j}\left(t-\tau_{2}(t)\right) \\
& +c_{3} \sum_{j=1}^{N} b_{i j} \Gamma \int_{t-r(t)}^{t} x_{j}(\xi) d \xi+u_{i}, \quad i=1,2, \ldots, l,
\end{aligned}
$$

$$
\begin{aligned}
\dot{x}_{i}= & f\left(x_{i}(t)\right)+c_{1} \sum_{j=1}^{N} g_{i j} \Gamma x_{j}(t) \\
& +\beta(t) c_{2} \sum_{j=1}^{N} a_{i j} \Gamma x_{j}\left(t-\tau_{1}(t)\right) \\
& +(1-\beta(t)) c_{2} \sum_{j=1}^{N} a_{i j} \Gamma x_{j}\left(t-\tau_{2}(t)\right) \\
& +c_{3} \sum_{j=1}^{N} b_{i j} \Gamma \int_{t-r(t)}^{t} x_{j}(\xi) d \xi, \quad i=l+1, l+2, \ldots, N,
\end{aligned}
$$

where $u_{i}=-c_{1} d_{i} \Gamma\left(x_{i}(t)-s(t)\right), \dot{d}_{i}=q_{i}\left(x_{i}(t)-s(t)\right)^{T} \Gamma\left(x_{i}(t)-\right.$ $s(t)), q_{i}>0, i=1,2, \ldots, l$.

Let $e_{i}(t)=x_{i}(t)-s(t)$ be the synchronization error. It is easy to obtain the following error dynamics:

$$
\begin{aligned}
\dot{e}_{i}= & f\left(x_{i}(t)\right)-f(s(t))+c_{1} \sum_{j=1}^{N} g_{i j} \Gamma e_{j}(t) \\
& +\beta(t) c_{2} \sum_{j=1}^{N} a_{i j} \Gamma e_{j}\left(t-\tau_{1}(t)\right) \\
& +(1-\beta(t)) c_{2} \sum_{j=1}^{N} a_{i j} \Gamma e_{j}\left(t-\tau_{2}(t)\right) \\
& +c_{3} \sum_{j=1}^{N} b_{i j} \Gamma \int_{t-r(t)}^{t} e_{j}(\xi) d \xi-c_{1} d_{i} \Gamma e_{i}(t), \\
\dot{e}_{i}= & f\left(x_{i}(t)\right)-f(s(t))+c_{1} \sum_{j=1}^{N} g_{i j} \Gamma e_{j}(t) \\
& +\beta(t) c_{2} \sum_{j=1}^{N} a_{i j} \Gamma e_{j}\left(t-\tau_{1}(t)\right) \\
& +(1-\beta(t)) c_{2} \sum_{j=1}^{N} a_{i j} \Gamma e_{j}\left(t-\tau_{2}(t)\right) \\
& +c_{3} \sum_{j=1}^{N} b_{i j} \Gamma \int_{t-r(t)}^{t} e_{j}(\xi) d \xi, \quad i=l+1, l+2, \ldots, N .
\end{aligned}
$$

We are now in a position to introduce the notion of synchronization in mean square sense for network (5).

Definition 3. The complex network (5) is said to be globally synchronized in mean square sense if $\lim _{t \rightarrow \infty} E\left\{\left\|e_{i}(t)\right\|^{2}\right\}=0$, $i=1,2, \ldots, N$, holds for any initial values.

Before ending this section, some assumptions and lemmas are given as follows. 
Assumption 4. There exist constants $\mu_{1}$ and $\mu_{2}$ such that $0 \leq$ $\dot{\tau}_{1}(t) \leq \mu_{1}<1$ and $0 \leq \dot{\tau}_{2}(t) \leq \mu_{2}<1$.

Assumption 5 (see [25]). There exists a constant $\theta>0$, such that the nonlinear function $f$ in (1) satisfies

$$
\begin{array}{r}
(x-y)^{T}(f(x)-f(y)) \leq \theta(x-y)^{T} \Gamma(x-y), \\
\forall x, y \in R^{n},
\end{array}
$$

where $\Gamma$ is the same as inner coupling matrix in network (1).

Lemma 6 (see [25] (Schur complement)). The linear matrix inequality

$$
\left[\begin{array}{ll}
Q(x) & S(x) \\
S(x)^{T} & R(x)
\end{array}\right]<0
$$

where $Q(x)=Q(x)^{T}$ and $R(x)=R(x)^{T}$, is equivalent to one of the following conditions:

(I) $Q(x)<0, R(x)-S(x)^{T} Q(x)^{-1} S(x)<0$;

(II) $R(x)<0, Q(x)-S(x) R(x)^{-1} S(x)^{T}<0$.

Lemma 7 (see [25]). Assume that $A, B$ are $N$ by $N$ Hermitian matrices. Let $\alpha_{1} \geq \alpha_{2} \geq \cdots \geq \alpha_{N}, \beta_{1} \geq \beta_{2} \geq \cdots \geq \beta_{N}$, and $\gamma_{1} \geq \gamma_{2} \geq \cdots \geq \gamma_{N}$ be eigenvalues of $A, B$, and $A+B$, respectively. Then, one has $\alpha_{i}+\beta_{N} \leq \gamma_{i} \leq \alpha_{i}+\beta_{1}, i=$ $1,2, \ldots, N$.

Lemma 8 (see [32]). For any positive symmetric constant matrix $Z=Z^{T}>0$, scalar $\gamma>0$, and vector function $\omega:[0, \gamma] \rightarrow R^{n}$ such that the integrations in the following are well defined, then one has

$$
\gamma \int_{0}^{\gamma} \omega^{T}(s) Z \omega(s) d s \geq\left(\int_{0}^{\gamma} \omega(s) d s\right)^{T} Z\left(\int_{0}^{\gamma} \omega(s) d s\right) .
$$

\section{Main Results}

In this section, we will investigate the stability criteria for the pinning controlled error system in mean square sense and give some low-dimensional conditions to guarantee that the network can achieve synchronization under the pinning scheme. Before giving the main results, for the sake of presentation simplicity, we denote

$$
\begin{aligned}
\rho= & \theta \\
& +\left(\frac{1}{2} c_{2}\left(\frac{\beta_{0}}{\left(1-\mu_{1}\right)}+\frac{\left(1-\beta_{0}\right)}{\left(1-\mu_{2}\right)}\right)+\frac{1}{2} c_{2} \lambda_{\max }(P)\right. \\
& \left.\quad+\frac{1}{2} c_{3} \lambda_{\max }(Q)+\frac{c_{3}}{2} r^{2}\right) \\
& \times\left(\lambda_{\min }(\Gamma)\right)^{-1},
\end{aligned}
$$

$\rho_{1}=\theta$

$$
\begin{aligned}
& +\left(\frac{1}{2\left(1-\mu_{1}\right)} c_{2}+\frac{1}{2} c_{2} \lambda_{\max }(P)+\frac{1}{2} c_{3} \lambda_{\max }(Q)+\frac{c_{3}}{2} r^{2}\right) \\
& \times\left(\lambda_{\min }(\Gamma)\right)^{-1}
\end{aligned}
$$

$\rho_{2}=\theta$

$$
\begin{aligned}
& +\left(\frac{1}{2} c_{2}\left(\frac{\beta_{0}}{\left(1-\mu_{1}\right)}+\frac{\left(1-\beta_{0}\right)}{\left(1-\mu_{2}\right)}\right)+\frac{1}{2} c_{2} \lambda_{\max }(P)\right) \\
& \times\left(\lambda_{\min }(\Gamma)\right)^{-1}
\end{aligned}
$$

where $P=\left(A A^{T}\right) \otimes\left(\Gamma \Gamma^{T}\right)$ and $Q=\left(B B^{T}\right) \otimes\left(\Gamma \Gamma^{T}\right)$.

Theorem 9. Suppose that Assumptions 1-5 hold; the pinning controlled network (7) globally asymptotically synchronizes to trajectory (6) in mean square sense if

$$
\lambda_{\max }\left(\left(\frac{1}{2}\left(G+G^{T}\right)\right)\right)<-\frac{\rho}{c_{1}} .
$$

Proof. Construct the following Lyapunov functional candidate:

$$
V(t)=V_{1}(t)+V_{2}(t)+V_{3}(t),
$$

where

$$
V_{1}(t)=\frac{1}{2} \sum_{i=1}^{N} e_{i}^{T}(t) e_{i}(t)+\sum_{i=1}^{l} \frac{c_{1}}{2 q_{i}}\left(d_{i}-d_{i}^{*}\right)^{2}
$$

in which $d_{i}^{*}>0$ are constants to be determined below, and

$$
\begin{aligned}
V_{2}(t)= & \frac{c_{2} \beta_{0}}{2\left(1-\mu_{1}\right)} \sum_{i=1}^{N} \int_{t-\tau_{1}(t)}^{t} e_{i}^{T}(\theta) e_{i}(\theta) d \theta \\
& +\frac{c_{2}\left(1-\beta_{0}\right)}{2\left(1-\mu_{2}\right)} \sum_{i=1}^{N} \int_{t-\tau_{2}(t)}^{t} e_{i}^{T}(\theta) e_{i}(\theta) d \theta \\
V_{3}(t)= & \frac{1}{2} c_{3} r \sum_{i=1}^{N} \int_{-r}^{0} \int_{t+\theta}^{t} e_{i}^{T}(\xi) e_{i}(\xi) d \xi d \theta
\end{aligned}
$$

Let $L$ be the weak infinitesimal generator of the random process along system (8). Then, we have

$$
\begin{aligned}
E\left\{L V_{1}(t)\right\}=\sum_{i=1}^{N} e_{i}^{T}(t)[ & g\left(e_{i}(t)\right)+c_{1} \sum_{j=1}^{N} g_{i j} \Gamma e_{j}(t) \\
& +c_{3} \sum_{j=1}^{N} b_{i j} \Gamma \int_{t-r(t)}^{t} e_{j}(\xi) d \xi \\
& +\beta_{0} c_{2} \sum_{j=1}^{N} w_{i j} \Gamma e_{j}\left(t-\tau_{1}(t)\right) \\
& +\left(1-\beta_{0}\right) c_{2} \\
& \left.\times \sum_{j=1}^{N} w_{i j} \Gamma e_{j}\left(t-\tau_{2}(t)\right)\right]
\end{aligned}
$$




$$
\begin{aligned}
& -\sum_{i=1}^{l} c_{1} d_{i} e_{i}^{T}(t) \Gamma e_{i}(t) \\
& +\sum_{i=1}^{l} c_{1}\left(d_{i}-d_{i}^{*}\right) e_{i}^{T}(t) \Gamma e_{i}(t), \\
E\left\{V_{2}(t)\right\}= & \frac{c_{2} \beta_{0}}{2\left(1-\mu_{1}\right)} \sum_{i=1}^{N} e_{i}^{T}(t) e_{i}(t) \\
& +\frac{c_{2}\left(1-\beta_{0}\right)}{2\left(1-\mu_{2}\right)} \sum_{i=1}^{N} e_{i}^{T}(t) e_{i}(t) \\
E\left\{L V_{3}(t)\right\}= & \frac{1}{2} c_{3} r^{2} \sum_{i=1}^{N} e_{i}^{T}(t) \Gamma e_{i}(t) \\
& -\frac{c_{2} \beta_{0}\left(1-\dot{\tau}_{1}(t)\right)}{2\left(1-\mu_{1}\right)} \\
& \times \sum_{i=1}^{N} e_{i}^{T}\left(t-\tau_{1}(t)\right) e_{i}\left(t-\tau_{1}(t)\right) \\
& -\frac{c_{2}\left(1-\beta_{0}\right)\left(1-\dot{\tau}_{2}(t)\right)}{2\left(1-\mu_{2}\right)} \\
& \times \sum_{i=1}^{N} e_{i}^{T}\left(t-\tau_{2}(t)\right) e_{i}\left(t-\tau_{2}(t)\right) \\
& \\
& \\
& \\
& \\
&
\end{aligned}
$$

Define $e(t)=\left(e_{1}^{T}(t), e_{2}^{T}(t), \ldots, e_{N}^{T}(t)\right)^{T}, D=\operatorname{diag}\left(d_{1}^{*}, \ldots\right.$, $d_{l}^{*}, \underbrace{0, \ldots}_{N-1,0}$. Note the fact that the inequality $2 x^{T} y \leq x^{T} M x+$ $y^{T} M^{-1} y$ holds for arbitrary $x, y \in R^{n N}$ and a positive definite matrix $M \in R^{n N \times n N}$. Then, recalling Assumption 5 and using Kronecker product technique, one has

$$
\begin{aligned}
E\{L & \left.V_{1}(t)\right\} \\
\leq & e^{T}(t)\left(\theta I_{N} \otimes \Gamma\right) e(t)+c_{1} e^{T}(t)(G \otimes \Gamma) e(t) \\
& \quad-c_{1} e^{T}(t)(D \otimes \Gamma) e(t) \\
& +c_{3} e^{T}(t)(B \otimes \Gamma) \int_{t-r(t)}^{t} e(\xi) d \xi \\
& +\beta_{0} c_{2} e^{T}(t)(A \otimes \Gamma) e\left(t-\tau_{1}(t)\right) \\
& +\left(1-\beta_{0}\right) c_{2} e^{T}(t)(A \otimes \Gamma) e\left(t-\tau_{2}(t)\right)
\end{aligned}
$$

$$
\begin{aligned}
\leq & e^{T}(t)\left(\theta I_{N} \otimes \Gamma\right) e(t)+c_{1} e^{T}(t)(G \otimes \Gamma) e(t) \\
& -c_{1} e^{T}(t)(D \otimes \Gamma) e(t) \\
& +\frac{1}{2} c_{2} e^{T}(t) P e(t)+\frac{1}{2} \beta_{0} c_{2} e^{T}\left(t-\tau_{1}(t)\right) e\left(t-\tau_{1}(t)\right) \\
& +\frac{1}{2}\left(1-\beta_{0}\right) c_{2} e^{T}\left(t-\tau_{2}(t)\right) e\left(t-\tau_{2}(t)\right) \\
& +\frac{1}{2} c_{3} e^{T}(t) Q e(t) \\
& +\frac{1}{2} c_{3}\left(\int_{t-r(t)}^{t} e(\xi) d \xi\right)^{T}\left(\int_{t-r(t)}^{t} e(\xi) d \xi\right)
\end{aligned}
$$

In view of Assumption 4, we get

$$
\begin{aligned}
E\left\{L V_{2}(t)\right\} \leq & \left(\frac{c_{2} \beta_{0}}{2\left(1-\mu_{1}\right)}+\frac{c_{2}\left(1-\beta_{0}\right)}{2\left(1-\mu_{2}\right)}\right) e^{T}(t) e(t) \\
& -\frac{c_{2} \beta_{0}}{2} e^{T}\left(t-\tau_{1}(t)\right) e\left(t-\tau_{1}(t)\right) \\
& -\frac{c_{2}\left(1-\beta_{0}\right)}{2} e^{T}\left(t-\tau_{2}(t)\right) e\left(t-\tau_{2}(t)\right) .
\end{aligned}
$$

By using Lemma 8, we obtain

$$
\begin{aligned}
E\left\{L V_{3}(t)\right\}= & \frac{1}{2} c_{3} r^{2} e^{T}(t) e(t) \\
& -\frac{1}{2} c_{3} r \int_{t-r}^{t} e^{T}(\xi) e(\xi) d \xi \\
\leq & \frac{1}{2} c_{3} r^{2} e^{T}(t) e(t)-\frac{1}{2} c_{3} r \int_{t-r(t)}^{t} e^{T}(\xi) e(\xi) d \xi \\
\leq & \frac{1}{2} c_{3} r^{2} e^{T}(t) e(t) \\
& -\frac{1}{2} c_{3}\left(\int_{t-r(t)}^{t} e(\xi) d \xi\right)^{T}\left(\int_{t-r(t)}^{t} e(\xi) d \xi\right)
\end{aligned}
$$

According to (19)-(22), we have

$$
\begin{aligned}
E\{L V(t)\} & \\
\leq & e^{T}(t)\left(\theta I_{N} \otimes \Gamma\right) e(t)+c_{1} e^{T}(t)(G \otimes \Gamma) e(t) \\
& -c_{1} e^{T}(t)(D \otimes \Gamma) e(t)+\frac{1}{2} c_{2} e^{T}(t) P e(t) \\
& +\frac{1}{2} c_{32} e^{T}(t) Q e(t) \\
& +\left(\frac{c_{2} \beta_{0}}{2\left(1-\mu_{1}\right)}+\frac{c_{2}\left(1-\beta_{0}\right)}{2\left(1-\mu_{2}\right)}\right) e^{T}(t) e(t) \\
& +\frac{1}{2} c_{3} r^{2} e^{T}(t) e(t) .
\end{aligned}
$$


It is easy to see that $P$ and $Q$ are symmetric, so we have $e^{T}(t) P e(t) \leq \lambda_{\max }(P) e^{T}(t) e(t)$ and $e^{T}(t) Q e(t) \leq$ $\lambda_{\max }(Q) e^{T}(t) e(t)$. Therefore, we get

$$
E\{L V(t, e(t))\} \leq e^{T}(t)\left(\left(M-c_{1} D\right) \otimes \Gamma\right) e(t),
$$

where $M=\rho I_{N}+(1 / 2) \mathcal{c}_{1}\left(G+G^{T}\right)$. It is obvious that matrix $M$ is symmetric. By using the matrix decomposition technique, we have $M-c_{1} D=\left[\begin{array}{cc}M_{1}-c_{1} D^{*} & M_{2} \\ M_{2}^{T} & M_{l}\end{array}\right]$, where $M_{1}$ and $M_{2}$ are matrices with appropriate dimensions, $D^{*}=$ $\operatorname{diag}\left(d_{1}^{*}, \ldots, d_{l}^{*}\right)$, and $M_{l}=\left(\rho I_{N}+c_{1}\left((1 / 2)\left(G+G^{T}\right)\right)\right)_{l}$ is the minor matrix of $M$ by removing its first $l$ row-column pairs. In view of (15) and Lemma 7, we have $\lambda_{\max }\left(M_{l}\right) \leq$ $\rho+c_{1} \lambda_{\max }\left(\left((1 / 2)\left(G+G^{T}\right)\right)_{l}\right)<0$, which implies that $M_{l}<0$. Here, if we choose some suitable positive constants $d_{i}^{*}>$ $\left(\lambda_{\max }\left(M_{1}-M_{2} M_{l}^{-1} M_{2}^{T}\right)\right) / c_{1}$, it follows from Lemma 6 that $M-c_{1} D<0$. In addition, since $\Gamma$ is a positive definite matrix, it is easy to see that $\left(M-c_{1} D\right) \otimes \Gamma<0$. It is clear that $E\{L V(t)\} \leq 0$, which implies that $\lim _{t \rightarrow \infty} E\left\{\left\|e_{i}(t)\right\|^{2}\right\}=0$. It follows from Definition 3 that the complex network (5) is synchronized with the isolated node (6) in mean square sense. This completes the proof.

Remark 10. Theorem 9 gives a low-dimensional sufficient condition to ensure pinning synchronization for complex network (5) with mixed probabilistic time-varying coupling delays. From Theorem 9, we can see that the network synchronization depends on seven basic elements: node dynamics $(\theta)$, coupling strength $\left(c_{1}, c_{2}\right.$, and $\left.c_{3}\right)$, network structure $(G, A$, and $B)$, inner coupling matrix $(\Gamma)$, the probability distribution of coupling delay $\left(\beta_{0}\right)$, the upper bound of distributed time delay $(r)$, and the derivative information of delay $\left(\mu_{1}, \mu_{2}\right)$. If the derived condition in Theorem 9 is satisfied, the synchronization can be achieved by pinning control small nodes.

Remark 11. Condition in (15) provides a criterion to determine the least number $l_{0}$ of pinned nodes for ensuring the network synchronization with fixed network structure, coupling strength, and pinning scheme. From (13), we have $c_{1}>-\rho / \lambda_{\max }\left(\left((1 / 2)\left(G+G^{T}\right)\right)_{l}\right)$, which gives a way to choose the appropriate coupling strength for network with fixed structure and pinning scheme. However, the theoretical value of $c_{1}$ is often much larger than that needed in practice. If $c_{1}$ is not large enough, it is not guaranteed that we can find a small fraction of network nodes such that pinning condition (15) holds. To achieve synchronization, we prefer to adopt the adaptive control approach to adjust the coupling strength, which can refer to [23].

Remark 12. It is worth pointing out that the considered model in (5) is different from the existing ones [26, 27], where only the deterministic coupling time delay was considered. Thus it is difficult to give some comparison with the existing results. In the next section, the effectiveness of the proposed method will be verified by some numerical examples.

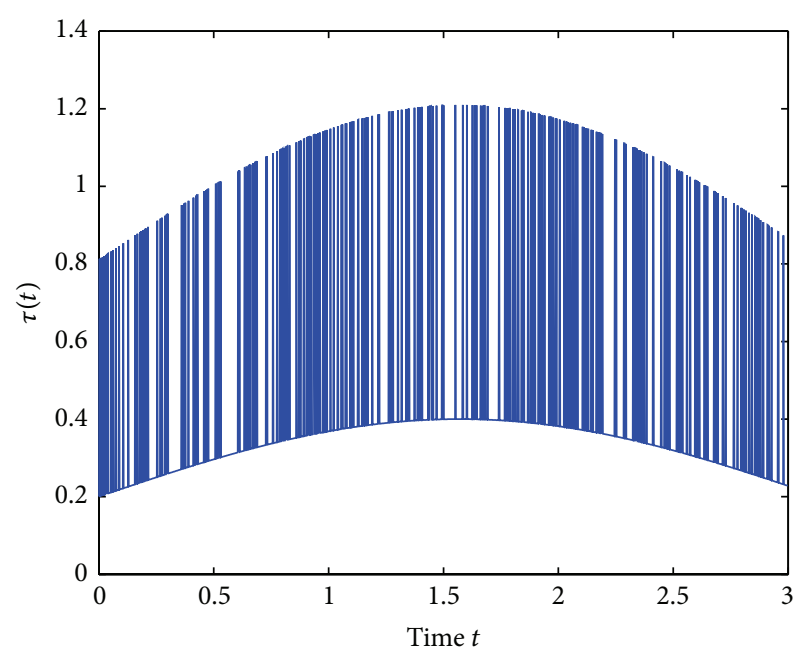

Figure 1: Random coupling delay $\tau(t)$.

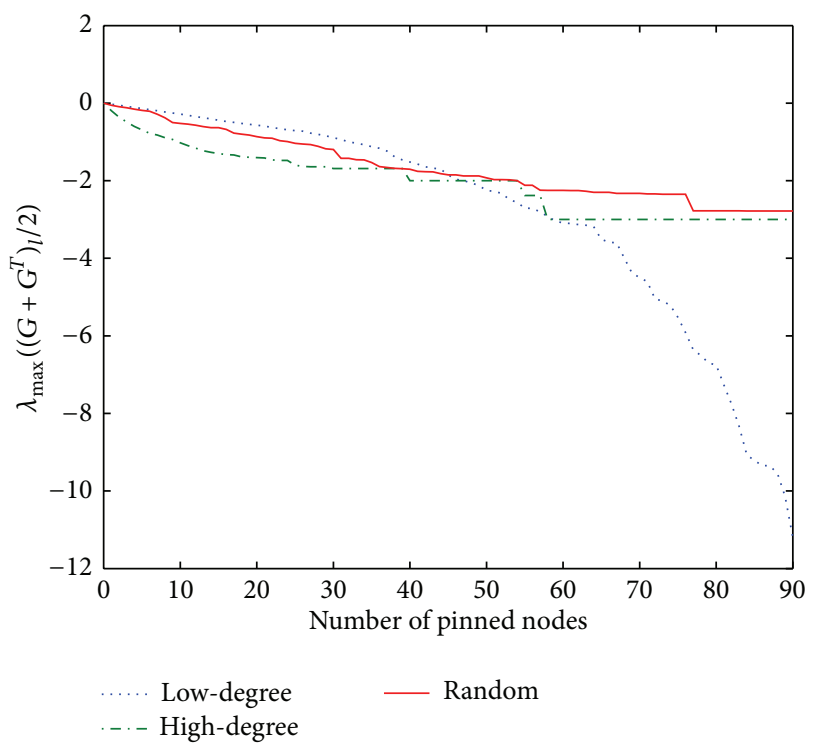

FIgURE 2: Orbits of $\lambda_{\max }\left(\left((1 / 2)\left(G+G^{T}\right)\right)_{l}\right)$ as functions of the number of pinned nodes by high-degree, low-degree, and random pinning schemes.

As a special case, when $\beta_{0}=1$ or $\beta_{0}=0$, the probabilistic coupling delay becomes the deterministic delay. Thus we have the following pinning controlled complex network model:

$$
\begin{aligned}
\dot{x}_{i}= & f\left(x_{i}(t)\right)+c_{1} \sum_{j=1}^{N} g_{i j} \Gamma x_{j}(t) \\
& +c_{2} \sum_{j=1}^{N} a_{i j} \Gamma x_{j}(t-\tau(t)) \\
& +c_{3} \sum_{j=1}^{N} b_{i j} \Gamma \int_{t-r(t)}^{t} x_{j}(\xi) d \xi \\
& -c_{1} d_{i} \Gamma\left(x_{i}(t)-s(t)\right),
\end{aligned}
$$




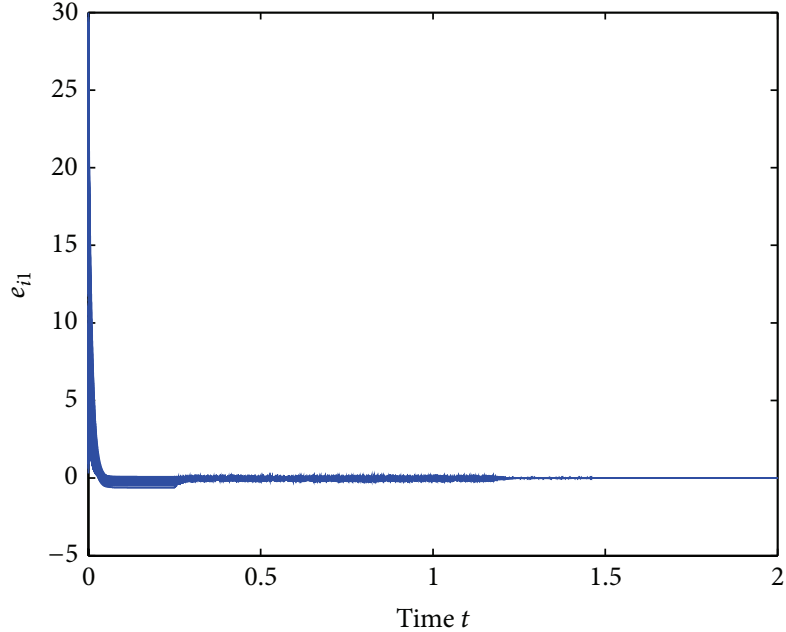

(a) $e_{i 1}(1 \leq i \leq 100)$

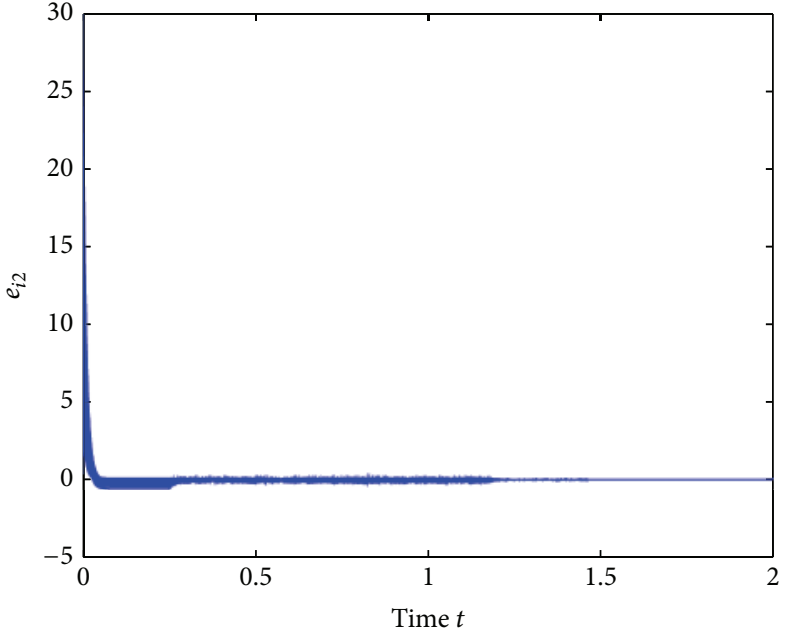

(b) $e_{i 2}(1 \leq i \leq 100)$

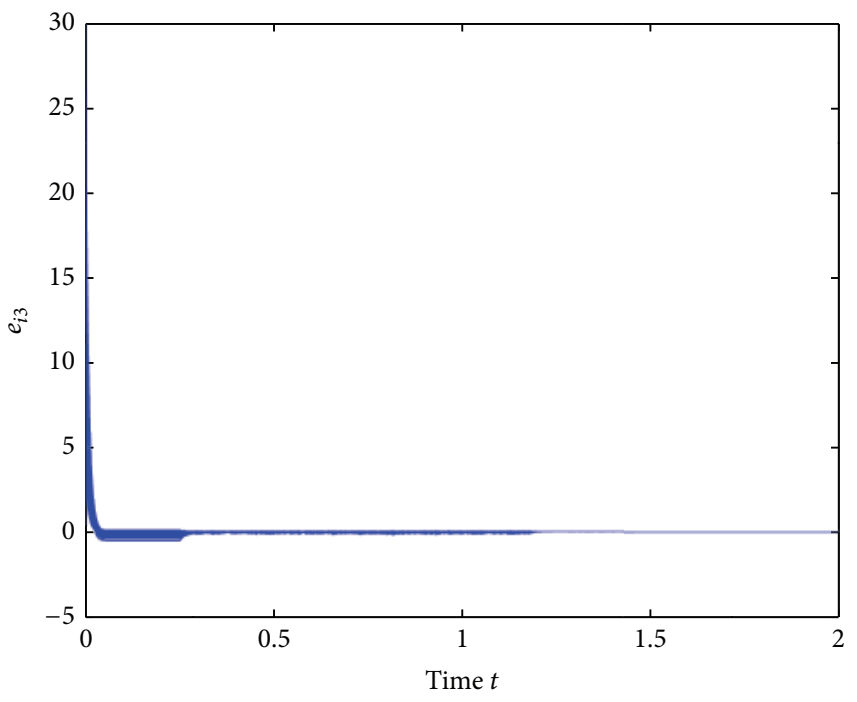

(c) $e_{i 3}(1 \leq i \leq 100)$

FIGURE 3: Synchronization errors $e_{i j}$ of the controlled network (5).

where $\dot{d}_{i}=q_{i}\left(x_{i}(t)-s(t)\right)^{T} \Gamma\left(x_{i}(t)-s(t)\right), q_{i}>0, i=1,2, \ldots, l$, and $d_{i}=0, i=l+1, \ldots, N$. According to Theorem 9, the following result is easily derived.

Corollary 13. Suppose Assumption 5 holds; the pinning controlled network (25) globally asymptotically synchronizes to trajectory (6) if

$$
\lambda_{\max }\left(\left(\frac{1}{2}\left(G+G^{T}\right)\right)_{l}\right)<-\frac{\rho_{1}}{c_{1}} .
$$

On the other hand, if there is no distributed coupling term in network model (1), that is, $B=0$, we have the following pinning controlled network model:

$$
\begin{aligned}
\dot{x}_{i}= & f\left(x_{i}(t)\right)+c_{1} \sum_{j=1}^{N} g_{i j} \Gamma x_{j}(t) \\
& +\beta(t) c_{2} \sum_{j=1}^{N} a_{i j} \Gamma x_{j}\left(t-\tau_{1}(t)\right)
\end{aligned}
$$

$$
\begin{aligned}
& +(1-\beta(t)) c_{2} \sum_{j=1}^{N} a_{i j} \Gamma x_{j}\left(t-\tau_{2}(t)\right) \\
& -c_{1} d_{i} \Gamma\left(x_{i}(t)-s(t)\right)
\end{aligned}
$$

where $\dot{d}_{i}=q_{i}\left(x_{i}(t)-s(t)\right)^{T} \Gamma\left(x_{i}(t)-s(t)\right), q_{i}>0, i=1,2, \ldots, l$, and $d_{i}=0, i=l+1, \ldots, N$. Based on Theorem 9, we have the following result.

Corollary 14. Suppose Assumption 5 holds; the pinning controlled network (27) globally asymptotically synchronizes to trajectory (6) in mean square sense if

$$
\lambda_{\max }\left(\left(\frac{1}{2}\left(G+G^{T}\right)\right)_{l}\right)<-\frac{\rho_{2}}{c_{1}} .
$$

Remark 15. It should be noted that the main result obtained in this paper can be extended to more general complex 


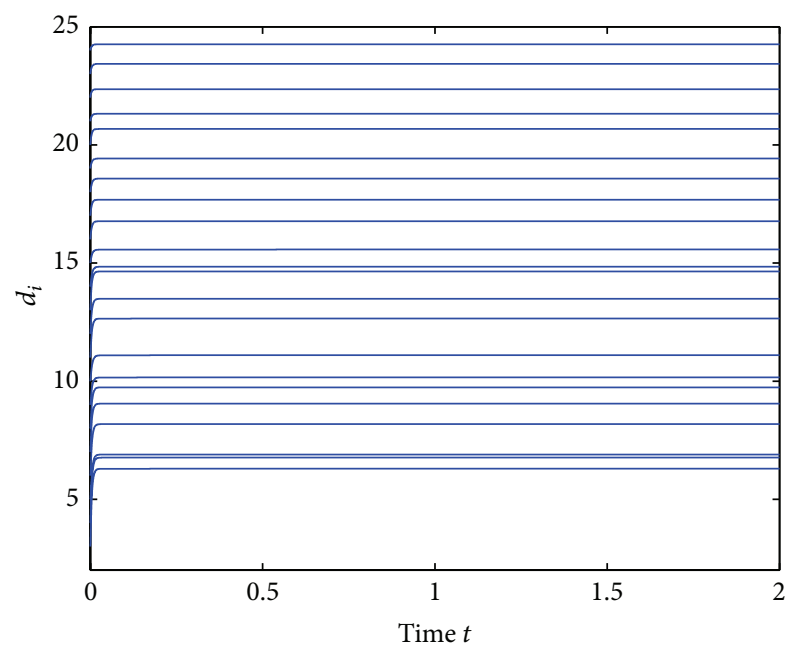

FIgURE 4: Evolution of adaptive feedback gains $d_{i}$ with $1 \leq i \leq 15$.

dynamical networks with delayed nodes, such as hybridcoupled delayed neural networks with mixed probabilistic time-varying delays.

\section{Numerical Examples}

In this section, a numerical example is used to verify the effectiveness of the proposed pinning synchronization criterion.

Here, we assume that the controlled network consists of 100 identical Chua systems. The dynamics at every node is described by

$$
f\left(x_{i}(t)\right)=\left\{\begin{array}{l}
\alpha\left(x_{i 2}(t)-x_{i 1}(t)-\phi\left(x_{i 1}(t)\right)\right) \\
x_{i 1}(t)-x_{i 2}(t)+x_{i 3}(t) \\
-\beta x_{i 2}(t),
\end{array}\right.
$$

where $\phi\left(x_{1}(t)\right)=b x_{1}(t)+(1 / 2)(a-b)\left(\left|x_{1}(t)+1\right|-\left|x_{1}(t)-1\right|\right)$ and $a=-1.27, b=-0.68, \alpha=10$, and $\beta=14.87$.

In addition, we assume that the coupling matrices $G$ and $A$ obey the scale-free distribution of the BA network with $m_{0}=m=3, N=100$, and the small-world model with the link probability $P=0.1, m=2, N=100$, respectively, and $B=0.5 \mathrm{~A}$. For simplicity, we set $\Gamma=\operatorname{diag}\{2,2,2\}, c_{1}=50$, $c_{2}=1, c_{3}=1$, and $\beta_{0}=0.8$. Let $\tau_{1}(t)=0.2+0.2 \sin (t)$ and $\tau_{1}(t)=0.81+0.4 \sin (t)$; then we get $\mu_{1}=0.2$ and $\mu_{2}=0.4$. Figure 1 depicts the random delay.

According to [29], we have $\theta=5.4263$. Then by some calculation, one has $\rho=-1.4270$. Here, the orbits of $\lambda_{\max }\left(\left((1 / 2)\left(G+G^{T}\right)\right)_{l}\right)$ as functions of the number of pinned nodes by high-degree, low-degree, and random pinning schemes are shown in Figure 2. It is obvious that the orbits decrease with the increase of pinning controlled nodes. We observe that one only needs 39,33 , and 22 nodes of network (5) to realize synchronization by using low-degree, random, and high-degree pinning schemes, respectively. Hence, it is better to use the high-degree pinning scheme in this case.

Now, we apply adaptive feedback control to the first 22 most highly connected nodes. In the numerical simulation, the initial values are given as follows: $d_{i}(0)=2+i$ and $q_{i}=2$ for $1 \leq i \leq 15, x_{i}(0)=(4+0.3 i, 5+0.3 i, 6+0.3 i)^{T}$, where $1 \leq i \leq 100$, and $s(0)=(4,5,6)^{T}$. The evolutions of the synchronization error and the pinning feedback gain are illustrated in Figures 3 and 4, respectively. Clearly, the synchronization for complex network (5) with probabilistic time delay and distributed time delay is achieved under the pinning scheme with $l=22$.

\section{Conclusion}

In this paper, the pinning synchronization problem has been investigated for a hybrid-coupled complex network with mixed probabilistic time-varying delays. The coupling configuration matrices are more general and not assumed to be symmetric or irreducible. A low-dimensional sufficient condition for the network synchronization by adding adaptive feedback controllers to a fraction of network nodes is presented. Finally, numerical simulation shows the effectiveness of the theoretical result.

\section{Conflict of Interests}

The author declares that there is no conflict of interests regarding the publication of this paper.

\section{Acknowledgments}

The work is supported by the National Natural Science Foundation of China (Grant nos. 61203049 and 61303020) and the Doctoral Startup Foundation of Taiyuan University of Science and Technology (Grant no. 20112010).

\section{References}

[1] T. Liu, J. Zhao, and D. J. Hill, "Exponential synchronization of complex delayed dynamical networks with switching topology," IEEE Transactions on Circuits and Systems I: Regular Papers, vol. 57, no. 11, pp. 2967-2980, 2010.

[2] X. Wu and H. Lu, "Exponential synchronization of weighted general delay coupled and non-delay coupled dynamical networks," Computers \& Mathematics with Applications, vol. 60, no. 8, pp. 2476-2487, 2010.

[3] Y. Wang, H. Zhang, X. Wang, and D. Yang, "Networked synchronization control of coupled dynamic networks with time-varying delay," IEEE Transactions on Systems, Man, and Cybernetics B Cybernetics, vol. 40, no. 6, pp. 1468-1479, 2010.

[4] Z.-X. Li, J. H. Park, and Z.-G. Wu, "Synchronization of complex networks with nonhomogeneous Markov jump topology," Nonlinear Dynamics, vol. 74, no. 1-2, pp. 65-75, 2013.

[5] T. H. Lee, J. H. Park, D. H. Ji, O. M. Kwon, and S. Lee, "Guaranteed cost synchronization of a complex dynamical network via dynamic feedback control," Applied Mathematics and Computation, vol. 218, no. 11, pp. 6469-6481, 2012.

[6] W. Zhong, J. D. Stefanovski, G. M. Dimirovski, and J. Zhao, "Decentralized control and synchronization of time-varying complex dynamical network," Kybernetika, vol. 45, no. 1, pp. 151$167,2009$. 
[7] J. Wu and L. Jiao, "Synchronization in complex dynamical networks with nonsymmetric coupling," Physica D, vol. 237, no. 19, pp. 2487-2498, 2008.

[8] J. Zhou, J. Lu, and J. Lv, "Adaptive synchronization of an uncertain complex dynamical network," IEEE Transactions on Automatic Control, vol. 51, no. 4, pp. 652-656, 2006.

[9] Y. Xu, W. Zhou, and J. Fang, "Adaptive synchronization of the complex dynamical network with double non-delayed and double delayed coupling," International Journal of Control, Automation and Systems, vol. 10, no. 2, pp. 415-420, 2012.

[10] L. Wang, Y. Jing, K. Zhi, and G. M. Dimirovski, "Adaptive exponential synchronization of uncertain complex dynamical networks with delay coupling," NeuroQuantology, vol. 6, no. 4, pp. 397-404, 2008.

[11] J. Lu, D. W. C. Ho, and J. Cao, "A unified synchronization criterion for impulsive dynamical networks," Automatica, vol. 46, no. 7, pp. 1215-1221, 2010.

[12] J. Tang and C. Huang, "Impulsive control and synchronization analysis of complex dynamical networks with non-delayed and delayed coupling," International Journal of Innovative Computing, Information and Control, vol. 11, pp. 4555-4564, 2013.

[13] J. Yao, H. O. Wang, Z. Guan, and W. Xu, "Passive stability and synchronization of complex spatio-temporal switching networks with time delays," Automatica, vol. 45, no. 7, pp. 1721$1728,2009$.

[14] Y. Liu and J. Zhao, "Generalized output synchronization of dynamical networks using output quasi-passivity," IEEE Transactions on Circuits and Systems I, vol. 59, no. 6, pp. 1290-1298, 2012.

[15] W. Xia and J. Cao, "Pinning synchronization of delayed dynamical networks via periodically intermittent control," Chaos, vol. 19, no. 1, Article ID 013120, 2009.

[16] S. Cai, Q. He, J. Hao, and Z. Liu, "Exponential synchronization of complex networks with nonidentical time-delayed dynamical nodes," Physics Letters A, vol. 374, no. 25, pp. 2539-2550, 2010.

[17] N. Li, Y. Zhang, J. Hu, and Z. Nie, "Synchronization for general complex dynamical networks with sampled-data," Neurocomputing, vol. 74, no. 5, pp. 805-811, 2011.

[18] Z. Wu, J. H. Park, H. Su, B. Song, and J. Chu, "Exponential synchronization for complex dynamical networks with sampleddata," Journal of the Franklin Institute, vol. 349, no. 9, pp. 27352749, 2012.

[19] J. Wang, "Synchronization of complex networks with random coupling strengths and mixed probabilistic time-varying coupling delays using sampled data," Abstract and Applied Analysis, vol. 2014, Article ID 845304, 12 pages, 2014.

[20] X. Li, X. Wang, and G. Chen, "Pinning a complex dynamical network to its equilibrium," IEEE Transactions on Circuits and Systems. I: Regular Papers, vol. 51, no. 10, pp. 2074-2087, 2004.

[21] T. Chen, X. Liu, and W. Lu, "Pinning complex networks by a single controller," IEEE Transactions on Circuits and Systems. I. Regular Papers, vol. 54, no. 6, pp. 1317-1326, 2007.

[22] J. Zhou, J. Lu, and J. Lü, "Pinning adaptive synchronization of a general complex dynamical network," Automatica, vol. 44, no. 4, pp. 996-1003, 2008.

[23] W. Yu, G. Chen, and J. Lv, "On pinning synchronization of complex dynamical networks," Automatica, vol. 45, no. 2, pp. 429-435, 2009.

[24] L. Deng, Z. Wu, and Q. Wu, "Pinning synchronization of complex network with non-derivative and derivative coupling," Nonlinear Dynamics, vol. 73, no. 1-2, pp. 775-782, 2013.
[25] Q. Song and J. Cao, "On pinning synchronization of directed and undirected complex dynamical networks," IEEE Transactions on Circuits and Systems I: Regular Papers, vol. 57, no. 3, pp. 672-680, 2010.

[26] Q. Song, J. Cao, and F. Liu, "Pinning-controlled synchronization of hybrid-coupled complex dynamical networks with mixed time-delays," International Journal of Robust and Nonlinear Control, vol. 22, no. 6, pp. 690-706, 2012.

[27] Y. Liang, X. Wang, and J. Eustace, "Adaptive synchronization in complex networks with non-delay and variable delay couplings via pinning control," Neurocomputing, vol. 123, pp. 292-298, 2014.

[28] J. Wang, R. Nie, and Z. Sun, "Pinning sampled-data synchronization for complex networks with probabilistic coupling delay," Chinese Physics B, vol. 23, Article ID 050509, 2014.

[29] Y. Wu, C. Li, A. Yang, and L. Song, "Pinning adaptive antisynchronization between two general complex dynamical networks with non-delayed and delayed coupling," Applied Mathematics and Computation, vol. 218, no. 14, pp. 7445-7452, 2012.

[30] Y. Zhang, D. Yue, and E. Tian, "Robust delay-distributiondependent stability of discrete-time stochastic neural networks with time-varying delay," Neurocomputing, vol. 72, pp. 12651273, 2008.

[31] X. Yang, J. Cao, and J. Lu, "Synchronization of coupled neural networks with random coupling strengths and mixed probabilistic time-varying delays," International Journal of Robust and Nonlinear Control, vol. 23, no. 18, pp. 2060-2081, 2013.

[32] K. Gu, "An integral inequality in the stability problem of time-delay systems," in Proceedings of the 39th IEEE Confernce on Decision and Control, pp. 2805-2810, Sydney, Australia, December 2000. 


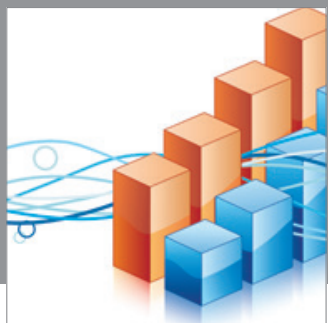

Advances in

Operations Research

mansans

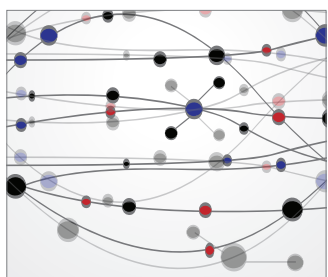

The Scientific World Journal
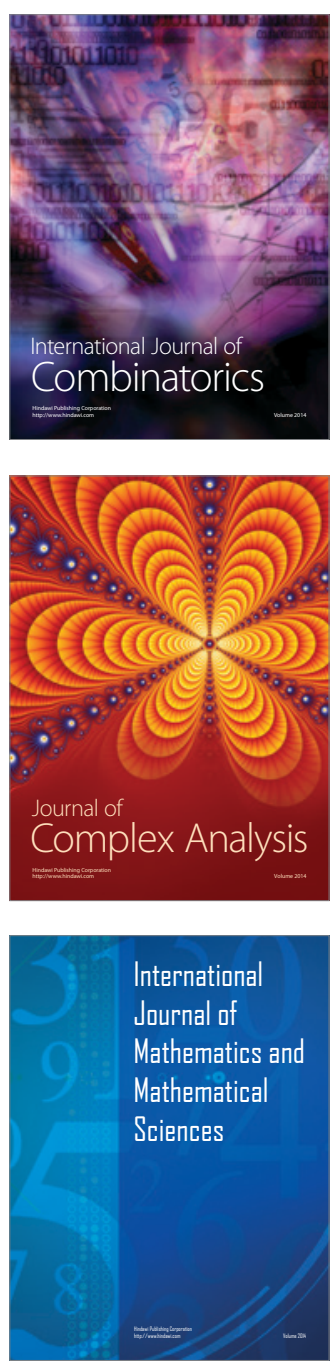
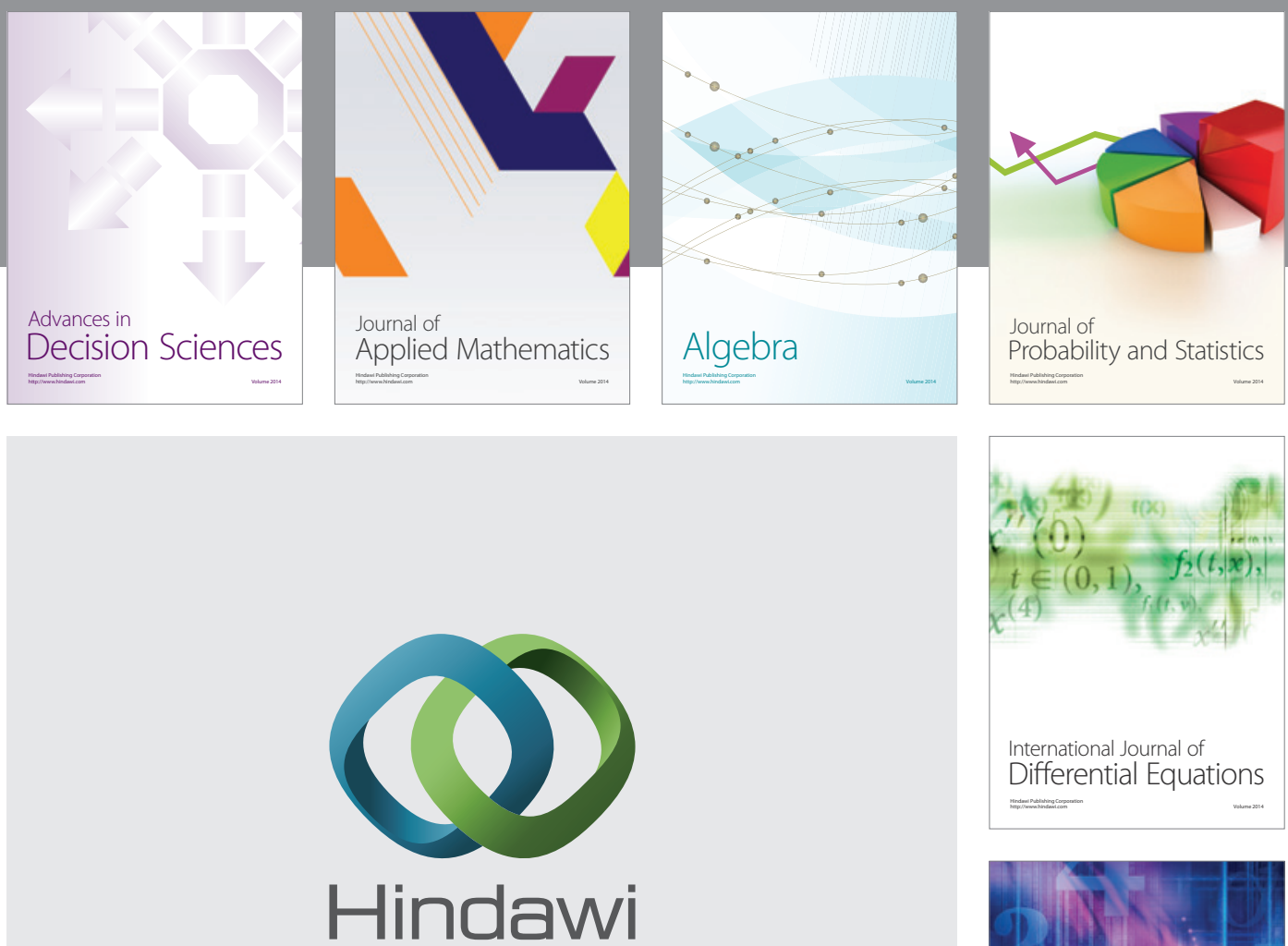

Submit your manuscripts at http://www.hindawi.com
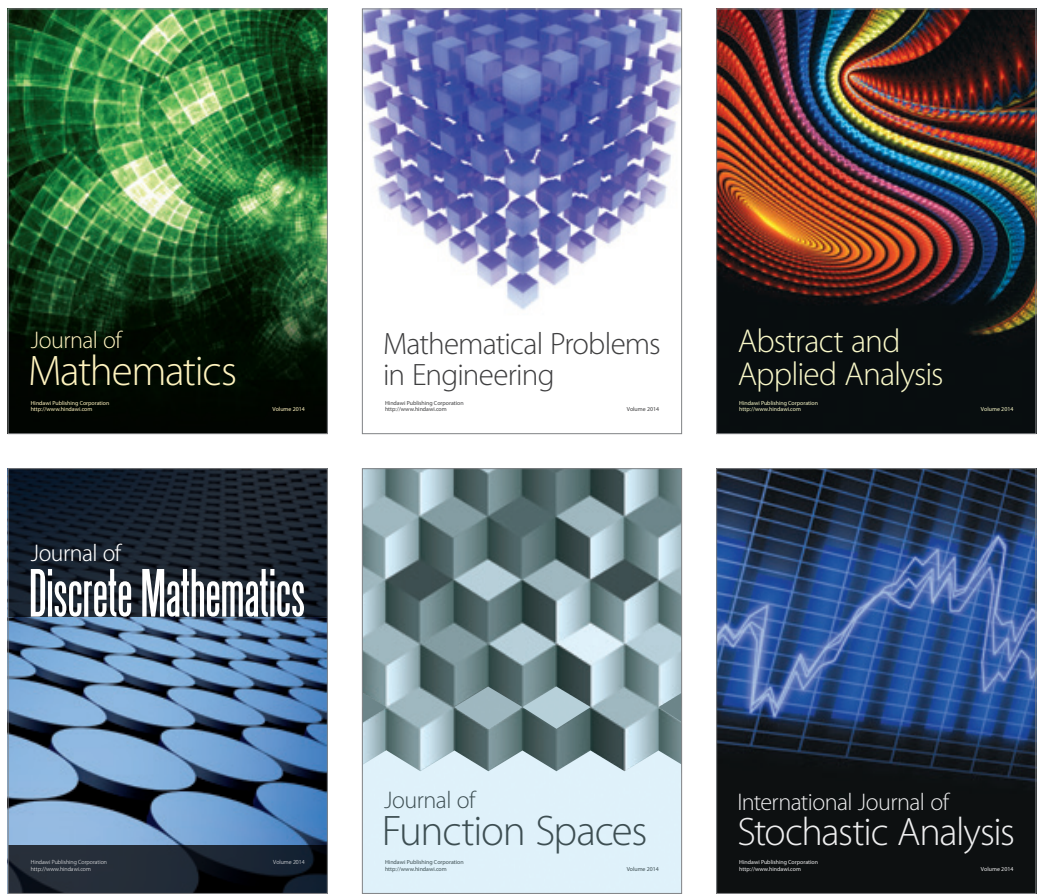

Journal of

Function Spaces

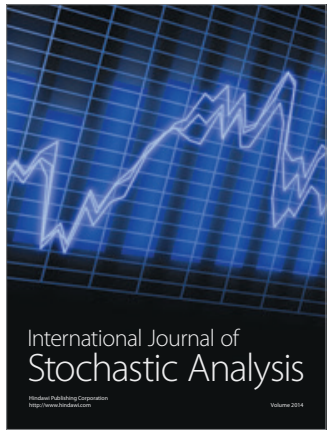

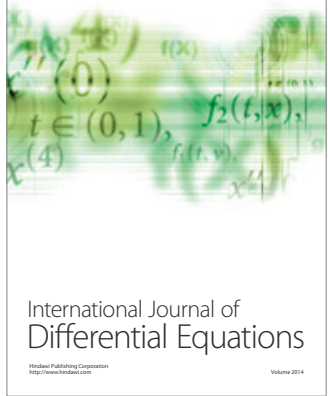
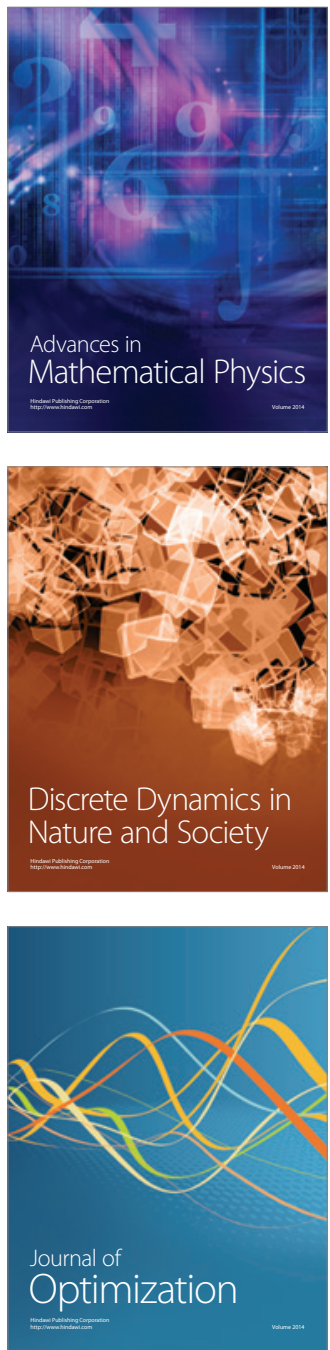